\title{
Importancia del reposo en los sanatorios para tuberculosos
}

\author{
Ignacio Duarte G. y Marcelo López C.
}

\section{The importance of rest in sanatoria for tuberculous patients}

Since ancient times the main indication for the treatment of pulmonary phthisis consisted in prescribing trips to better climates, horseback riding or walking outdoors. In the second half of the XIX ${ }^{\text {th }}$ century, the concept of sanatoria for TB therapy was developed in Europe, in order to confine patients in premises where abundant food, exposition to pure air and graded exercises were provided. In those places, rest progressively acquired more importance, becoming a thoroughly established ritual. In the $\mathrm{XX}^{\text {th }}$ century, the regulations for the recently founded Chilean sanatoriums incorporated European schedules. In the case of Hospital Sanatorio El Peral, the rules became progressively stricter until 1952, when permanent rest in bed was determined as the regime required. This rule could be explained by the policy of using this sanatorium for the treatment of more serious forms of the disease.

Key words: Tuberculosis, sanatoria, rest.

Palabras clave: Tuberculosis, sanatorios, reposo.
Pontificia Universidad Católica de Chile, Santiago.

Facultad de Medicina.

Programa de Estudios Médicos Humanísticos.

Recibido: 23 de enero 2009 Aceptado: 23 de marzo 2009

Correspondencia a: Ignacio Duarte G. iduarte@med.puc.cl
$\mathrm{E}$ n la segunda mitad del siglo XIX y en los primeros dos tercios del siglo XX el tratamiento de la tuberculosis llegó a estar centrado en el sanatorio, modelo institucional creado antes que se descubriera el bacilo de Koch en 1882, y que siguió vigente hasta la incorporación de la quimioterapia. En los sanatorios se practicaba la "cura dietético-higiénica", cuyos componentes básicos eran: la cura de aire, la cura de alimentación y la cura de reposo.

El presente trabajo tiene por objeto analizar la génesis y evolución de la indicación del reposo como componente del régimen sanatorial en general y sus características, según las disposiciones reglamentarias de algunos sanatorios chilenos.

\section{Rol creciente del reposo}

Desde tiempos antiguos, el tratamiento de elección para la tisis consistía en paseos o viajes al aire libre. Así, en el siglo I Celso indicaba:

"Es preciso, si las fuerzas del enfermo lo permiten, emprender larga navegación y cambiar de clima para encontrar un aire más denso que el del país del que se aleja... Tendrá el paciente que renunciar a los negocios, y a todo aquello que pueda ser motivo de preocupación, procurará dormir mucho... evitará catarros... indigestiones...mantendrá la cabeza cubierta y el cuello bien tapado... curará la tos... combatirá la fiebre".

En los siglos siguientes se insistió en los viajes en búsqueda de climas más benignos. Tomas Sydenham (1624-1689) prescribía a los tísicos largos paseos a caballo y Gehrard Van Swieten (1700-1772) recomendaba que aquellos sin recursos para viajar consiguieran trabajo como cocheros para desplazarse al aire libre $^{2}$. En el mismo siglo XVIII, William Buchan indicaba que, a las primeras señales de consunción, el paciente se trasladara al aire puro del campo, donde debía hacer todo el ejercicio que pudiera. Agregaba

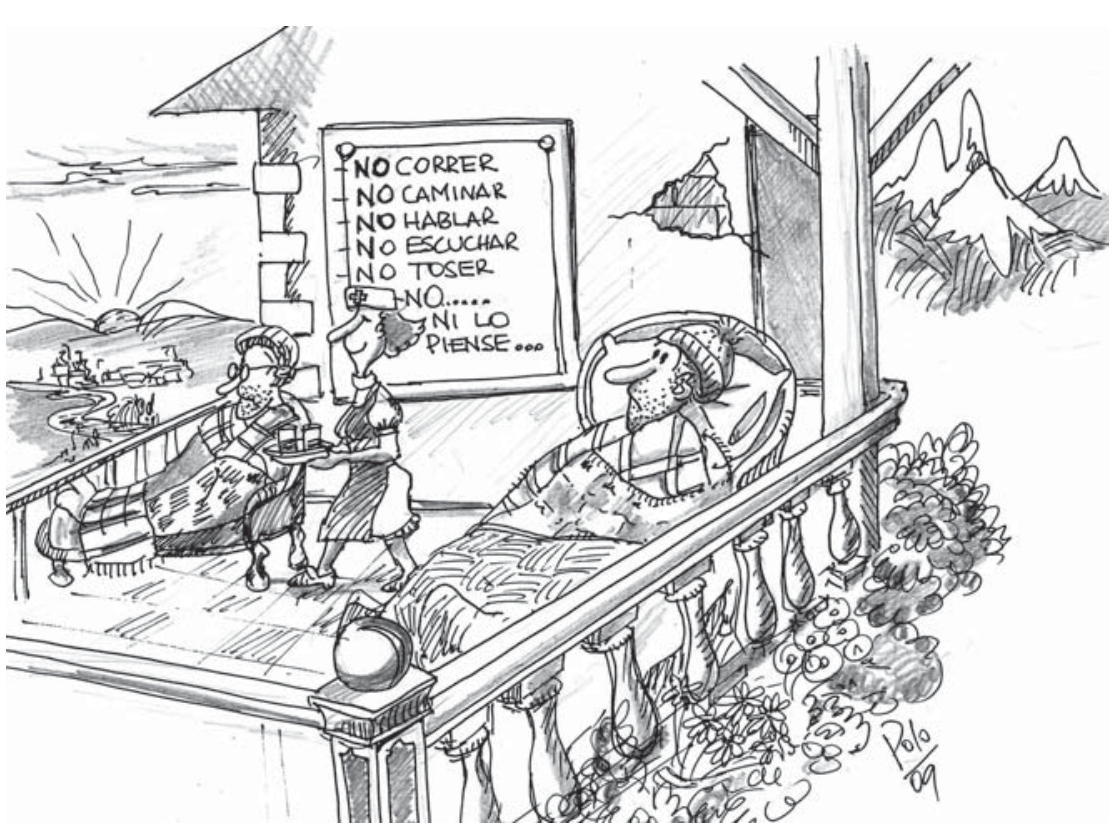


que "los que tengan espíritu y fuerzas para emprender un viaje largo pueden esperar gran ventaja de él. En mi inteligencia esto ha curado con mucha frecuencia una consunción cuando ya el enfermo estaba según todas las apariencias en el último estado de la enfermedad, y en que las medicinas no habian hecho ningún efecto. De aqui podemos inferir que si el viaje se hiciese á tiempo no dejaría de curarla." ${ }^{3,4}$.

En las primeras décadas del siglo XIX un Diccionario de Ciencias Médicas, redactado por "una sociedad de los más célebres profesores de Europa" recomendaba para la tisis pulmonar: dieta, respirar aire con temperatura moderada constante y movimiento moderado, este último indispensable para sostener la circulación y el equilibrio en las funciones. Se instaba a "evitar con cuidado las largas vigilias, los estudios inmoderados y todas las pasiones, y no olvidar que (los tísicos) son más sensibles e irritables que el común de los hombres. La cólera, poniendo toda la circulación en un vivo movimiento, fuerza el calibre de los vasos empujando la sangre hacia ellos en gran cantidad: de aqui nacen epistaxis y hemoptisis".

Agregaban que "Cuando cualquier órgano está predispuesto a padecer, debe ejercitarse con moderación y mantenerse en un estado de quietud, tanto mayor cuanto más grave sea su enfermedad". Ante signos de "sobre-escitacion en el pulmón, con predisposición á la hemoptisis,... imponer el más riguroso silencio, y evitar cualquier movimiento excesivo que pueda acelerar la circulación y respiración."

Acerca de la hemoptisis el Diccionario recomendaba "quietud absoluta, tranquilidad de espiritu, habitación ventilada; la cabeza elevada en la cama, los brazos cruzados sobre el pecho, y las piernas dobladas", a lo que debían agregarse sangrías, dietas y jarabes ${ }^{5}$.

A mediados del siglo XIX el médico inglés George Bodington preconizaba la creación de establecimientos para internar a los tuberculosos y someterlos al régimen de ejercicio, dieta, tratamiento general y estrecha vigilancia médica diaria en un medio rural elevado y seco, sin variaciones importantes de la temperatura, con una atmósfera libre de bruma y humedad. Allí debían hacer caminatas o cabalgar al aire libre en la mañana temprano hasta donde lo permitieran sus fuerzas: "La aplicación de aire puro a la superficie interna de los pulmones es el sedativo más poderoso que puede emplearse, y hace más para promover la curación y cierre de las cavidades y úlceras pulmonares que cualquier otro medio"'.

El médico prusiano Hermann Brehmer llegó a formarse las siguientes ideas sobre la tuberculosis pulmonar: era muy infrecuente en zonas montañosas de distintas partes del mundo; era potencialmente curable; su patogenia consistía en una nutrición pulmonar deficiente por un escaso aporte de sangre debido a un corazón débil y pequeño. Sobre estas bases formuló su plan terapéutico, que comprendía un programa de reposo en cama combinado con ejercicio en las alturas y alimentación abundante: por la menor presión atmosférica el individuo requeriría un incremento de su función cardíaca y se activaría su metabolismo ${ }^{7}$. Con este objetivo, creó en 1859 en Görbersdorf, Silesia, un establecimiento situado a alrededor de 500 metros de altitud, destinado a la cura de la tuberculosis, que es reconocido desde el punto de vista cronológico como el primero en su tipo ${ }^{8,9}$.

El doctor Peter Dettweiler que estuvo internado como paciente de Brehmer y después fue su ayudante, fundó en 1876 una institución similar en Falkenstein, cerca de Frankfurt. Más que en el ejercicio enérgico y el clima de altura, puso énfasis en la exposición al aire puro, control médico y educación más estrechos. El recurso terapéutico más importante fue el reposo en galerías techadas pero abiertas al aire, por hasta 10 horas diarias durante todas las estaciones del año: con ese objeto diseñó sillas reclinadas para que los enfermos pudieran estar cómodos. Dettweiler, que acuñó la palabra "Sanatorio" para su establecimiento, llegó a la conclusión de que la cura por reposo al aire libre fortalecía el organismo y se acomodaba cabalmente a enfermos de cualquier condición o estado de la enfermedad, e informaba que alrededor de $15 \%$ de sus pacientes eran dados de alta sin signos evidentes de enfermedad $^{10,11}$. Años después, el visitante podía ver en Falkenstein largas filas de pacientes reclinados en sus tumbonas ${ }^{12}$

Se consolidó así el concepto de sanatorio antituberculoso para la estancia prolongada de pacientes sometidos a un régimen disciplinado de reposo, alimentación, exposición al aire y ejercicio graduado en un lugar protegido y provisto de arboledas, jardines y senderos para caminar. A esto se fueron agregando procedimientos médico-quirúrgicos orientados al tratamiento de la afección pulmonar, como las inyecciones de cloruro de calcio, compuestos arsenicales y glicerina cresotada ${ }^{13}$ a los que se agregó la colapsoterapia. Siguiendo este modelo se construyeron numerosos establecimientos similares en diversos países, donde se eligieron emplazamientos variados: sanatorios de alta montaña, de altitud media, marítimos, y periféricos o suburbanos ${ }^{14}$

El reposo llegó a ser un ritual que incluía, entre otras cosas, prohibición de leer, escribir, conversar, incorporarse, escuchar radio y colocar los brazos detrás de la cabeza ${ }^{15}$. A esto se agregaba un complicado procedimiento para envolverse en frazadas para la permanencia al aire libre ${ }^{16}$ : 
"Se extendían las mantas, una después de otra, sobre el fondo de la silla, de tal manera que rebasasen bastante por los pies. Luego uno se tendía encima y se comenzaba por doblar la manta interior, primero en toda su longitud hasta los hombros, luego en su parte inferior por encima de los pies, sentándose y cogiendo el doblez de la manta, primero de un lado y luego del otro, y aplicando exactamente estos dos dobleces sobre el reborde de la chaise-longue, si se quería obtener la mayor regularidad posible. Se procedía luego de la misma manera con la manta exterior -era un poco más dificil de manejar".

En 1897, no existían aún sanatorios en Chile: el doctor Ernesto Soza explicaba en qué consistían estos establecimientos $^{17}$, y se refería al reposo en los siguientes términos:

"Jeneralmente el enfermo al ingresar al sanatorio, a consecuencia de las molestias del viaje, la impresión que le causa el establecimiento i sobre todo la acción estimulante del cambio de clima, le sobreviene un lijero agravamiento de sus dolencias; sobre todo la fiebre i la falta de apetito. Para combatir estos sintomas $i$ sobre todo mientras se acostumbran a un nuevo clima, se les tiene en su lecho uno o dos días, al cabo de los cuales, sistemáticamente, se les hace levantarse por unas dos horas, las cuales aprovechará el enfermo en la veranda de su pabellón, que es una galería ancha, abierta siempre al medio dia, en la cual tendrá una silla larga donde permanecerá acostado i con mucho abrigo, sin hacer el menor ejercicio. Cada día aumentará en una hora su estadía en la veranda, pero siempre deberá hacerlo con el consentimiento del médico...".

Carlos Díaz expresaba en 1910 que la vida del bronquítico debe ser enteramente de reposo, el que debe ser a la vez moral y físico: "nada de negocios, de trabajo, de inquietudes, la cama o la silla larga". Incluía el reposo sexual, dado que "el acto sexual, elevando la temperatura un grado aproximadamente, predispone a las hemoptisis"18.

En la Memoria para optar al grado de Licenciado en Medicina, atribuida a Félix Bulnes (1923) se extraen de la bibliografía extranjera los siguientes conceptos:

"La fatiga que aparece precozmente en la evolución de la tuberculosis, la inestabilidad de todas la funciones: vaso-motora, nerviosa, térmica, etc., constituyen todo un conjunto que coloca al tuberculoso en un estado extremadamente sensible a todo surmenage, tanto físico, como intelectual y moral. Esto traería una verdadera necesidad de reposo y sería ... la justificativa más acertada del método de cura.

Esta práctica sería además un factor de ahorro ya que suprime las principales fuentes del desgaste del organismo, como es el trabajo muscular por un lado e intelectual por el otro. Esta energía sería aprovechada para sostener con mayores probabilidades de éxito la lucha contra la infección tuberculosa".

Agregaba que el reposo, al evitar los movimientos del órgano enfermo, impide la congestión, disminuyendo la absorción de toxinas.

Con el objeto de obtener el máximo rendimiento de la cura de reposo, se detallaba que debía hacerse en forma metódica, continuada durante todo el tiempo que fuera necesario, abarcando las esferas: moral, intelectual, física y sexual. Destacaba la dificultad de "proporcionar al tuberculoso un reposo moral, dada su psicología y excitabilidad nerviosa. Deberían evitarse las emociones: el tuberculoso que quiera sanar debe hacer una vida monótona sin agitación, auste$r a$, sin inquietudes de ninguna especie, ni económicas, ni sentimentales, ni profesionales, etc.

El reposo intelectual debe ser completo, deben suprimirse toda clase de estudios, las lecturas deben ser muy bien seleccionadas y jamás se harán por un largo espacio de tiempo y aún en ciertos casos prohibirlas en absoluto... Se les aconsejará escribir lo menos posible".

En cuanto al reposo físico se distinguían tres grados:

- La cura de inmovilización, permaneciendo en cama y evitando los movimientos, indicada en los períodos congestivos y en los episodios de hemoptisis.

- La cura amortiguada, permaneciendo en cama, sin recomendación especial de inmovilización. Recetada "para los incidentes evolutivos ligeros, decaimiento, convalecencia, eretismo cardíaco, complicaciones intercurrentes, etc.".

- La cura de reposo en las galerías, régimen regular de los sanatorios, apropiado para las tuberculosis apiréticas o con pequeñas alzas de temperatura, en pacientes con buen estado general. Tres períodos prolongados de reposo cada día en chaise-longue en las galerías expuestas al aire, alternando con pequeños paseos que iban adaptándose progresivamente al estado de salud del enfermo ${ }^{19}$.

\section{El reposo en los reglamentos de sanatorios chilenos}

A partir del primer sanatorio creado en Chile en la ciudad de Los Andes en 1904, se procuraron seguir las pautas establecidas en Europa ${ }^{20}$. A tal grado llegó la identificación con el recurso básico de la vida nosocomial, que los pacientes internados llegaron a denominarse a sí mismos "los reposantes"21.

La primera norma escrita que hemos encontrado data de 1931: corresponde a la Casa de Salud Carolina 
Dousther de Tocornal, sanatorio privado ubicado en San José de Maipo. Fue redactado por su Director, el doctor Héctor Orrego Puelma. Se disponían tres sesiones de cura de reposo en galería ${ }^{22}$.

El mismo doctor Orrego Puelma publicó en 1937 un Reglamento de los Sanatorios de altura de la Caja del Seguro Obrero, en el que se leían, entre otras, las disposiciones que se transcriben:

"Las horas de reposo en las galerías, son obligatorias para todos los enfermos y nadie podrá disminuir su duración o cambiar su forma.

Durante la cura, los enfermos permanecerán tendidos en sus sillas de reposo, y deberán abstenerse de gritar, cantar o reir estrepitosamente para no turbar el orden.

Es permitido, durante la cura de reposo, leer o conversar en voz baja y con moderación a fin de evitar la tos.

Todo enfermo, debe permanecer cubierto en su silla de reposo, especialmente las extremidades inferiores, a fin de evitar enfriamientos por inmovilidad. $^{23}$.

Los sanatorios "El Peral" en Puente Alto y el de Putaendo, dependientes de la Junta de Beneficencia, se inauguraron en 1938 el primero ${ }^{24}$ y el segundo en 1940. En ambos se generaron sendos reglamentos, fechados respectivamente en 1938 y 1942. Los dos documentos son muy similares: en los párrafos correspondientes al reposo se establecía:

"Salvo enfermos que permanezcan en cama por prescripción médica, los reposos deben hacerse en las galerías dispuestas para este objeto.

Ningún enfermo podrá abandonar el reposorio antes de la hora indicada... Quedan prohibidas las levantadas por razones personales, debiendo los enfermos hacer sus menesteres particulares antes o después del reposo.

El $1^{\circ}$ y el 3er. reposo del día serán "Reposos relativos", es decir, que los enfermos podrán leer, conversar moderadamente, o desarrollar otras labores que no signifiquen esfuerzo, a condición que su cuerpo descanse totalmente sobre la cama y que no adopte posiciones forzadas.

El $2^{\circ}$ reposo, será "Reposo absoluto", en el cual están terminantemente prohibidas las conversaciones o actividad de cualquier especie... No se permiten los juegos (ajedrez, damas, etc.) durante estos repo$\operatorname{sos}{ }^{25,16}$.

Otra versión del reglamento para los enfermos del Hospital-Sanatorio "El Peral", publicada en 1943 contenía los mismos párrafos, a los que se incorporaba uno inicial que señalaba que todo enfermo hospitalizado debía permanecer constantemente en cama, hasta que su médico lo autorizara para levantarse: los otros acápites se aplicaban "a los enfermos que se levanten" 27.

En un nuevo Reglamento del mismo Hospital Sanatorio, fechado en 1952, las condiciones se tornaban más estrictas, como se indicaba en las frases iniciales del apartado "Del reposo sanatorial":

"Los enfermos hospitalizados en el Sanatorio "El Peral” están sometidos a un régimen de reposo... (que consistía en) la permanencia continua en cama". Sólo había obligación de levantarse en la mañana temprano para el aseo personal y necesidades fisiológicas imprescindibles. Las comidas, desayuno, almuerzo, once y cena se debían tomar en cama, con prohibición de escuchar radio. Después del almuerzo y la cena podían levantarse un corto rato para aseo y necesidades fisiológicas.

\section{Características y horarios del reposo}

- Reposo absoluto: “Enfermo en posición de cúbito dorsal (de espaldas)... los enfermos sólo están autorizados para leer y escuchar radio. Igualmente ocupaciones similares que no exijan esfuerzo alguno y que puedan ejecutarse con las manos, sin levantar la cabeza de la almohada ni abandonar, por ningún motivo, la posición de cúbito dorsal. Queda prohibido levantarse para cumplir necesidades fisiológicas". Esta modalidad regía de 9 a 12, 13 a 15 (siesta), 15 a 15:30, 17:45 a 18:45, 20 a 21. A las 21 horas, silencio absoluto. Se permitía oír " $L a$ Familia Chilena" y, debidamente autorizados, transmisiones radiales deportivas extraordinarias.

Reposo relativo: “... los enfermos podrán escribir. Los pacientes que están autorizados por el médico, podrán realizar algunas labores manuales (dibujar, cartonaje, etc) que no signifiquen esfuerzo. En este lapso los enfermos están autorizados para usar el almohadón, pero de ninguna manera a levantarse de la cama". El horario asignado era de 15:45 a 17:30 horas $^{28}$.

El reglamento de 1952 implicaba, pues, un nuevo enfoque del reposo en los enfermos hospitalizados, desde un régimen habitual como los de 1938 y 1943 -en que los internados se levantaban diariamente, tomaban sus comidas en el comedor y sus reposos de cura de aire en galerías- hasta las nuevas disposiciones de permanencia continua en cama.

La explicación de este cambio parece encontrarse en un nuevo enfoque de los objetivos del Establecimiento.

En efecto, en la Segunda Reunión Anual de Sanatorios efectuada en 1943 se había concluido que los sanatorios chilenos debían dedicarse exclusivamente al tratamiento y no al diagnóstico inicial del enfermo, 
con el objeto de optimizar los recursos invertidos. En este mismo sentido, se estimaba que deberían recibir sólo casos de tuberculosis probadamente activas y con no menos de $50 \%$ de probabilidades de recuperación $^{29}$.

Por otra parte, en 1944 los médicos de El Peral habían asumido la definición de Hospital-Sanatorio como "el instituto de asistencia al tuberculoso más completo en organización y dotación, con sus equipos médicos, quirúrgicos y de especialistas que pueden ofrecer al paciente, a la par que la cura de reposo, la terapéutica activa de la colapsoterapia médica y quirúrgica y de las complicaciones de la tuberculosis $" 30$. Esto implicaría reservar para los Sanatorios -instituciones de menor complejidad- a los enfermos que tuvieran indicación de reposo exclusivo y para el Hospital-Sanatorio los casos con indicación de colapso médico complicado o tratamiento quirúrgico.

El punto de vista indicado determinó que el HospitalSanatorio El Peral decidiera emplear sus camas, "las más caras de la República" para el tratamiento integral de las formas de afección pulmonar más seria y avanzada, en lugar de destinar esfuerzos para casos como pleuresías o nódulos pulmonares de ninguna o escasa actividad. Al reseñar la actividad desplegada en los 10 primeros años de funcionamiento, el doctor René García Valenzuela, Director del nosocomio, informó que se había empezado a seleccionar cada día más a los enfermos acreedores a una terapéutica activa por la extensión de sus lesiones y lo incierto de su porvenir. La nueva política significó aumentar el número de camas de 360 a 660, reforzar el laboratorio clínico y crear, no sin dificultades, un Servicio de Cirugía Torácica ${ }^{31}$.

\section{Se impone la quimioterapia}

En el mundo, la era de los sanatorios con su tríada de reposo, alimentación y aire puro llegó a su fin a comienzos de la segunda mitad del siglo XX. En efecto, después del descubrimiento del bacilo de Koch comenzó una búsqueda de sustancias que pudieran inhibirlo, lo que sólo se obtuvo en el siglo XX con la incorporación de la estreptomicina, el ácido paraaminosalicílico (PAS) y la isoniacida ${ }^{32}$. En 1948, una investigación señera multicéntrica randomizada en pacientes de sanatorios demostró la superioridad de agregar estreptomicina al simple régimen higieno-dietéti$\mathrm{co}^{33}$. Por otra parte, en 1966 se publicó el seguimiento a cinco años de un ensayo de terapia con isoniazida y PAS durante un año en Madrás (India), comparándose el efecto en enfermos internados en sanatorios con el obtenido en pacientes pobres que siguieron habitando en sus hogares, sometidos a la sub-alimentación, el hacinamiento y una penosa actividad laboral: no se encontraron diferencias significativas en la evolución de ambos grupos ${ }^{34,35}$. Se anunciaba así la consolidación de la quimioterapia anti-tuberculosa administrada en el seno de la comunidad, que se demostró más efectiva, rápida y con una relación costo-beneficio considerablemente superior que la de aquellos establecimientos donde los pacientes permanecían largos meses o años con resultados insatisfactorios ${ }^{36,37}$ que se manifestaban en recaídas y reingreso sanatorial en hasta la mitad de los casos.

Pasada la mitad del siglo XX, los sanatorios chilenos se fueron consolidando como hospitales generales o destinados a otras diversas funciones. Actualmente, la administración del tratamiento quimioterápico de la tuberculosis es preferentemente ambulatoria ${ }^{38}$.

\section{Resumen}

Desde la antigüedad, la indicación principal para el tratamiento de la tisis pulmonar consistía en prescribir viajes hacia climas más benignos, cabalgar o caminar al aire libre. En la segunda mitad del siglo XIX se desarrolló en Europa en concepto de sanatorios para la terapia de la tuberculosis (TBC), con el objeto de confinar a los pacientes en un establecimiento que les proporcionaba alimentación abundante, aire puro y ejercicios físicos graduales. En esos lugares adquirió progresivamente mayor importancia el reposo, que se convirtió en un ritual minuciosamente establecido. En el siglo XX los reglamentos de los sanatorios chilenos, recientemente fundados, incorporaron las disposiciones europeas. En el caso del Hospital Sanatorio El Peral, el reglamento se hizo progresivamente más estricto hasta determinar en 1952 que el régimen habitual era la permanencia de los pacientes en cama. Esta norma podría explicarse por la política de destinar dicho establecimiento al tratamiento de formas graves de la enfermedad.

\section{Referencias}

1.- Celso A C. Los ocho libros de la medicina. Libro tercero p. 105-166. Barcelona: Editorial Iberia 1966.

2.- Dubos R, Dubos J. The white plague. capítulo 11: Treatment and natural resistance. New Brunswick, New Jersey; Rutgers University Press; 1996, p. 139-53.

3.- Buchan W. De la consunción. En: Medicina Doméstica. Séptima edición. Capítulo XVIII. París: Librería de Garnier Hermanos; 1890, p. 140-51.

4.- Duarte I, Chuaqui C. Ideas sobre la consunción en un libro de medicina doméstica del siglo XVIII. Ars Médica 2007; 14: 113-26.

5.- Diccionario de Ciencias Médicas, Tomo 36. Tisis. Madrid: Imprenta de Dom Mateo Repullés; 1826, p. 
295-353.

6.- Keers R Y. Two forgotten pioneers: James Carson and Gerorge Bodington. Thorax 1980; 35: 483-9.

7.- Knopf A. The centenary of Brehmer's birth. Am Rev Tuberc 1926; 14: 207-10.

8.- Davis A L. History of the sanatorium movement. En: W. N. Rom, S. Garay (Eds.): Tuberculosis. Boston: Little Brown and Company; 1996, p. 35-54.

9.- Duarte I. Sanatorios para tuberculosos: auge y decadencia. Ars Médica 2005; 11: 203-18.

10.- Kinghorn H M. Brehmer and Dettweiler: a review of their methods of treatment of pulmonary tuberculosis. Am Rev Tuberc 1922; 5: 950-72.

11.- Öchsner-Pischel M. Erfinder der Freiluftliegekur - Peter Dettweiler und die Lungenheilanstalt in Falkenstein im Taunus. Pneumologie 2005; 59: 349-53.

12.- Bowditch V M. Medical reminiscences: I. Visits to Brehmer's and Dettweiler's sanatoria. J Outdoor Life 1919; 16: 65-71.

13.- Deformes E. Anotaciones sobre la Acción Antituberculosa en Chile especialmente en Valparaíso. Presentado al Congreso Internacional Americano de Medicina e Higiene en Buenos Aires el 25 de mayo. Valparaíso: Sociedad Imprenta y Litografía Universo; 1910 .

14.- Sauret J. La cura sanatorial de la tuberculosis. Enf Emerg 2001; 3: 199-205.

15.- Ayvazian L F. History of tuberculosis. En: L. B. Reichman, E. S. Hershfield (Eds.): Tuberculosis; a comprehensive international approach. Capítulo 1. New York: Marcel Decker, Inc.; 1993, p. 1-20.

16. - Mann T. La montaña mágica. Santiago de Chile: Ediciones Ercilla; 1945, p. 140-1.

17. - Soza E. Sanatorio para tuberculosos; base científica i práctica de la secuestración de los tuberculosos en estos establecimientos bajo su doble fin de su tratamiento hijiénico i de la defensa social. Santiago de Chile: Imprenta Cervantes; 1897.

18.- Díaz C. Sanatorios para tuberculosos. Memoria de prueba para optar al grado de licenciado en la Facultad de Medicina y Farmacia de la Universidad de Chile, 1910.

19.- Bulnes F. Ensayo de cura dietético-higiénica en el tratamiento de la tuberculosis pulmonar. Memoria de prueba para optar al grado de licenciado en la Facultad de Medicina y Farmacia de la Universidad de Chile. Santiago de Chile 1923.

20.- Duarte I, López M. Sanatorios para tuberculosos en Chile: primeros establecimientos (1886-1920). An Chil Hist Med 2006; 16: 211-24.

21. - Lara A. Un centro de readaptación hace falta en Santiago. Laennec 1940; 8: 4.

22.- Orrego H. Reglamento de la Casa de Salud "Carolina
Dousther de Tocornal". San José de Maipo 1931.

23. - Reglamento de los Servicios de Tuberculosis de la Caja del Seguro Obligatorio. Título IX: De los Sanatorios de Altura. Ap Resp Tuberculosis 1937; II: 84-94.

24,- López M. Un plan científico para combatir la peste blanca: origen del Sanatorio El Peral, 1927-1938. An Chil Hist Med 2006; 16: 57-84.

25. - Hospital-Sanatorio para broncopulmonares "El Peral". Reglamento. Santiago de Chile: Imprenta Universitaria 1938.

26.- Servicios de Beneficencia y Asistencia Social. Reglamento del Sanatorio de Putaendo. Santiago de Chile: Talleres Gráficos Casa Nacional del Niño; 1942.

27. - Hospital-Sanatorio para broncopulmonares "El Peral". Reglamento (Para los enfermos). Santiago de Chile: Imprenta Universitaria; 1943.

28. - Hospital-Sanatorio para broncopulmonares "El Peral". Reglamento para el régimen interno de los enfermos. Santiago de Chile: Imprenta Universitaria; 1952.

29.- Segunda Reunión Anual de los Sanatorios Antituberculosos. Conclusiones. Valparaíso; 1943.

30.- Río S, Orrego H, Alonso A. Crítica al tratamiento de la tuberculosis pulmonar en Chile. Tercer Congreso PanAmericano de Tuberculosis Montevideo, 1934.

31.- García Valenzuela R. Breve síntesis de un decenio de labor sanatorial. Boletín del Hospital-Sanatorio "El Peral". 1948; VIII: 6-11.

32.- Ledermann W. La tuberculosis después del descubrimiento de Koch. Rev Chil Infect. Edición Aniversario 2003: 48-50.

33.- Medical Research Council. Streptomycin treatment of pulmonary tuberculosis: a Medical Research Council investigation. Br Med J 1948; ii 4582-j.

34.- Tuberculosis Chemotherapy Centre. Madras: a concurrent comparison of home and sanatorium treatment of pulmonary tuberculosis in South India. Bull World Health Org 1959; 21: 51-131.

35.- Dawson J J, Devadatta S, Fox W, Radhakrishna S, Ramakrishnan C V, Somasundaram P R, et al. A 5-year study of patients with pulmonary tuberculosis in a concurrent comparison of home and sanatorium treatment for one year with isoniazide plus PAS. Bull World Health Org 1966; 34: 533-51.

36.- Goldstein S E. After the sanatorium what? J Outdoor Life 1914; 11: 266-8.

37. - Weisner DE. Sanatorium follow up studies. Am Rev Tuberc 1922; 6: 320-326.

38. - Ministerio de Salud de Chile. Programa nacional de control de la tuberculosis, 2005, p. 81. http:// www.redsalud.gov.cl/archivos/TUBERCULOSIS.pdf. (accedido el 5 de noviembre de 2008). 